Check for updates

Cite this: Phys. Chem. Chem. Phys., 2020, 22, 22289

Received 29th July 2020

Accepted 31st August 2020

DOI: 10.1039/d0cp04228e

rsc.li/pccp

\title{
Probing the location of the unpaired electron in spin-orbit changing collisions of NO with Art
}

\author{
Cornelia G. Heid, ${ }^{a}$ Imogen P. Bentham, ${ }^{a}$ Victoria Walpole, $\neq^{\mathrm{a}}$ Razvan Gheorghe, ${ }^{a}$ \\ Pablo G. Jambrina, (D) b F. Javier Aoiz (D) ${ }^{c}$ and Mark Brouard (D) *a
}

\begin{abstract}
Understanding the molecular forces that drive a reaction or scattering process lies at the heart of molecular dynamics. Here, we present a combined experimental and theoretical study of the spin-orbit changing scattering dynamics of oriented NO molecules with Ar atoms. Using our crossed molecular beam apparatus, we have recorded velocity-map ion images and extracted differential and integral cross sections of the scattering process in the side-on geometry. We observe an overall preference for collisions close to the $\mathrm{N}$ atom in the spin-orbit changing manifold, which is a direct consequence of the location of the unpaired electron on the potential energy surface. In addition, a prominent forward scattered feature is observed for intermediate, even rotational transitions when the atom approaches the molecule from the O-end. The appearance of this peak originates from an attractive well on the $A^{\prime}$ potential energy surface, which efficiently directs high impact parameter trajectories towards the region of high unpaired electron density near the $\mathrm{N}$-end of the molecule. The ability to orient molecules prior to collision, both experimentally and theoretically, allows us to sample different regions of the potential energy surface(s) and unveil the associated collision pathways.
\end{abstract}

\section{Introduction}

To capture and elucidate collisional and reactive processes between molecules accurately and to a high level of detail is one of the biggest goals in chemical dynamics. In its pursuit, a joint effort between experiment and theory is in most cases indispensable. Calculations are needed, for instance, to assign transitions in high-resolution spectra, ${ }^{1,2}$ to verify product distributions of reactions, ${ }^{3-5}$ or to rationalize resonances observed in scattering experiments. ${ }^{6-11}$ Experiments, in turn, are required to test calculated potential energy surfaces (PESs) ${ }^{12-18}$ and to validate theoretical predictions ${ }^{19-21}$ and approximations. ${ }^{2-24}$

Increasingly refined methods to control molecules in collisions and reactions have provided particularly sensitive probes for testing the accuracy of PESs. Recent work by Vogels et al., has challenged the accuracy of the PESs involved in the scattering of NO with para- $\mathrm{H}_{2} \cdot{ }^{25}$ In their experiments, in which the velocity of the NO molecules was controlled by a Stark decelerator and the

\footnotetext{
${ }^{a}$ Department of Chemistry, University of Oxford, The Chemistry Research Laboratory, 12 Mansfield Road, Oxford, OX1 3TA, UK. E-mail: mark.brouard@chem.ox.ac.uk

${ }^{b}$ Departamento de Quimica Física, Universidad de Salamanca, 37008, Salamanca, Spain

${ }^{c}$ Departamento de Química Física, Facultad de Química, Universidad Complutense, 28040 Madrid, Spain

$\dagger$ Electronic supplementary information (ESI) available. See DOI: 10.1039/d0cp04228e ‡ Current address: Max Planck Institute for Biophysical Chemistry, Am Faßberg 11, 37077 Göttingen, Germany.
}

$\mathrm{H}_{2}$ molecules were slowed down via an 'anti-seeding' technique with $\mathrm{Ne}$, they were able to resolve a Feshbach resonance around $14 \mathrm{~cm}^{-1}$. Comparison with the position of the resonance calculated on two very similar high-level coupled-cluster PESs, ${ }^{26,27}$ allowed to establish the more accurate of the two potentials, highlighting the sensitivity of their experimental approach. Likewise, measurements of the reaction rate of metastable $\mathrm{He}$ with $\mathrm{H}_{2}$ and its isotopologues ${ }^{16,18,28}$ showed that shape (or orbiting) resonances observed below $10 \mathrm{~K}$ could be used to benchmark quantum dynamics calculations to within $7 \times 10^{-3} \mathrm{~cm}^{-1} \cdot{ }^{18}$ Observations of resonances in other high-resolution experiments of molecular interactions, including in vibrational excitation studies, ${ }^{8,11}$ have demonstrated similar sensitivities to the corresponding calculated energy levels and PESs. ${ }^{5-7,9,14,29}$

In addition to probing the local energy landscape via dynamical resonances, initial alignment ${ }^{23,30-38}$ or orientation ${ }^{39-44}$ of selected reactant quantum states can be used to gauge the geometric preferences and uncover the associated reaction pathways of molecular encounters. ${ }^{37,38}$ In this case, specific portions of the PES can be explored selectively by confining the relative geometry of the interacting species. Spatial alignment (alignment parallel or perpendicular to a reference axis) through polarized laser light has revealed pronounced preferences for specific initial configurations, ${ }^{30,33-38}$ and even enabled the three-dimensional visualization of distinct microscopic reaction pathways. ${ }^{23}$

Molecular orientation, in which a specific end or side of a molecule can be directed towards a collision partner, is most 
widely achieved through hexapole state selection coupled with adiabatic passage into a static electric ${ }^{40-42,45,46}$ or magnetic ${ }^{43,44,47}$ field. The good agreement between experimental and calculated steric preferences obtained in studies of inelastic collisions of state selected $\mathrm{NO}(\mathrm{X})$ with $\mathrm{Ar}$ and $\mathrm{He}$ atoms ${ }^{40-42,48-51}$ have confirmed the accuracy of the $\mathrm{NO}(\mathrm{X})+\mathrm{He} / \mathrm{Ar}$ ground electronic PESs, ${ }^{52-54}$ while (unoriented) scattering experiments of electronically excited $\mathrm{NO}(\mathrm{A})$ with $\mathrm{Ne}^{17}$ pointed to shortcomings in the calculated PESs. ${ }^{55,56}$

Electric field orientation requires the molecule to be oriented to posses a permanent dipole moment and, if only the dominant first-order Stark effect is taken into account, to be open-shell. ${ }^{57}$ The open-shell nature of the ground ${ }^{2} \Pi$ state of these molecules leads to two spin-orbit manifolds, given by the sum of the projections of the electronic orbital $(\Lambda)$ and spin $(\Sigma)$ angular momenta onto the internuclear axis. For the particular case of the extensively studied ${ }^{2} \Pi$ diatomic NO, the values of $\Lambda$ and $\Sigma$ are \pm 1 and $\pm 1 / 2$, respectively; the spin-orbit quantum number, $\Omega$, can thus take on values of $|\Omega|=1 / 2$ and $|\Omega|=3 / 2$. The former corresponds to the spin-orbit ground state, and the latter corresponds to the spin-orbit excited state, which lies $123 \mathrm{~cm}^{-1}$ above the $|\Omega|=1 / 2$ state. ${ }^{58}$ Depending on the collision energy, rotational excitations are possible to either of the two manifolds.

Integral and differential scattering cross sections have been measured for a range of (oriented and unoriented) open-shell molecules and collision partners, in particular $\mathrm{OH}^{46,59-63}$ and NO. ${ }^{64-67}$ The $\mathrm{OH}+\mathrm{Rg}(\mathrm{Rg}=$ rare gas $)$ systems have been systematically studied by Scharfenberg et al., ${ }^{62}$ who showed that the contribution of the spin-orbit excited states to the total scattering intensity significantly decreased with increasing polarizability and mass of the atom. This observation was in part correlated with the larger anisotropy and deeper attractive wells in the potentials for the heavier rare gases, suggesting that the more repulsive character of the PESs for the lighter atoms is conducive to spin-orbit excitations, which require an additional $\sim 140 \mathrm{~cm}^{-1}$ (relative to the spin-orbit conserving manifold) to be accessed. ${ }^{68}$ Consistent with this picture of a more impulsive, lower impact parameter collision are the more backward scattered differential cross sections measured for spin-orbit excited NO after collisions with $\mathrm{Ar}, \mathrm{Kr}$, and Xe. ${ }^{66,69-73}$

In the present work, we focus on transitions from the rotational and spin-orbit ground state to the spin-orbit excited manifold, $|j=1 / 2,| \Omega|=1 / 2\rangle \rightarrow\left|j^{\prime},\right| \Omega^{\prime}|=3 / 2\rangle, \quad$ in the scattering of oriented NO molecules with Ar, using a combination of experiment and theory to unravel the dynamics. In a first step, we compare the experimental data obtained in the side-on orientation with full QM calculations to establish very good agreement between the experimental and computational results. In the second step, we then simulate the experiments at the limit of infinite field orientation, again using a QM treatment. The calculated differential and integral cross sections provide new insights into the molecular forces that promote spin-orbit changing excitations. By controlling the initial relative orientation of the collision partners, we are able to probe selected parts of the potential energy surface; the resulting trends in steric preference, as a function of $\Delta j=j^{\prime}-j$ (where $j$ and $j^{\prime}$ designate the initial and final rotational states, respectively), will be shown to be particularly sensitive to the location of the unpaired electron within the NO molecule.

\section{Methods}

\section{A. Experimental methods}

The experimental setup is identical to the one described previously for detection of the spin-orbit conserving $(\Delta \Omega=0)$ rotational transitions. ${ }^{50,51}$ The NO molecules were expanded in a skimmed molecular beam containing about 15\% NO in Ar. Using a hexapole, the molecules were state selected in their lowfield seeking $f \Lambda$-doublet state ${ }^{64}$ and focused into the center of the scattering chamber where they were crossed with a neat argon beam at right angles. Prior to collision with the Ar atoms, the NO were adiabatically oriented in a static electric field located in the interaction region.

In the electric field, the initially pure $f \Lambda$-doublet state evolves into a superposition state with contributions from both $\Lambda$-doublets: ${ }^{40,48,74}$

$$
\left|j m_{E}\right| \Omega|E\rangle=\frac{1}{\sqrt{2}}\left[\alpha(E)\left|j m_{E}\right| \Omega|e\rangle+\beta(E)\left|j m_{E}\right| \Omega|f\rangle\right] .
$$

Here, $j$ is the rotational quantum number and $m_{E}$ its projection onto the electric field vector $\boldsymbol{E}, \Omega$ is the spin-orbit quantum number $(\Lambda+\Sigma)$, and $\alpha(E)$ and $\beta(E)$ are the field-dependent mixing parameters, such that $\alpha^{2}+\beta^{2}=2 .{ }^{40,48}$ At the electric field strength of the experiment, the mixing parameters are $|\alpha|=0.64$ and $|\beta|=1.26$, yielding an about $2: 1$ ratio between initial $f$ and $e$ states. (At infinite field, $|\alpha|=|\beta|=1$.)

The field was oriented perpendicular to the relative velocity vector $\left(\boldsymbol{k}=\boldsymbol{v}_{\mathrm{Ar}}-\boldsymbol{v}_{\mathrm{NO}}\right)$ such that the Ar preferentially impacted on the side of the diatomic (see Fig. 1(a) and (b)). By switching the direction of the electric field every 200 laser shots, images for both side-on orientations were collected alternatingly. The spin-orbit rotationally excited $(\Delta \Omega=1)$ NO molecules were ionized by a $\left(1+1^{\prime}\right)$ Resonance Enhanced Multiphoton Ionization (REMPI) process ${ }^{75}$ with the resonant laser pulse $(\approx 226 \mathrm{~nm})$ coming from a dye laser, pumped by an excimer laser and tuned to a specific $\mathrm{NO}(A) \leftarrow \mathrm{NO}(X)$ transition on the mixed $Q_{21}+R_{11}$ branch. The ionization pulse was provided by the $308 \mathrm{~nm}$ fundamental of the $\mathrm{XeCl}$ excimer laser. The nascent NO ions were subsequently velocity mapped ${ }^{76}$ onto a dual $\mathrm{MCP} /$ phosphor screen detector and imaged $^{77}$ with an intensified charge-coupled device camera connected to a data acquisition computer. The laser beams and the NO molecular beam were operated at $10 \mathrm{~Hz}$, while the Ar beam was operated at $5 \mathrm{~Hz}$ to allow for background subtraction on a shot-to-shot basis.

The DCSs for the two orientations of each of the measured spin-orbit excited transitions were extracted by fitting a linear combination of modified spherical harmonics basis functions to the sum and difference of the images for the two sides. From the fitted images, the relevant polarization moments were extracted and then used to calculate the DCSs. The method is described in detail in our earlier work ${ }^{50,51}$ (see in particular Section 4 in ref. 51). 


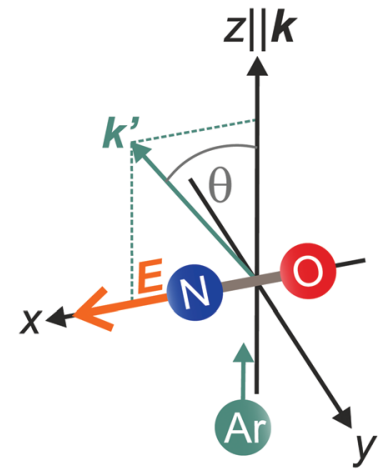

a) $+x$

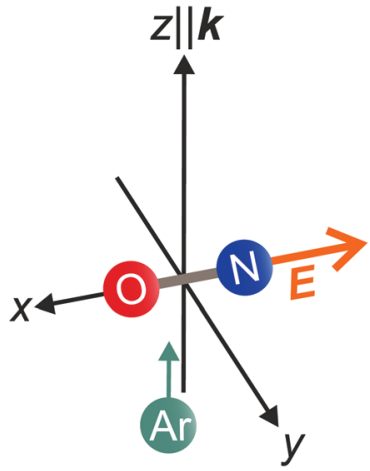

b) $-x$
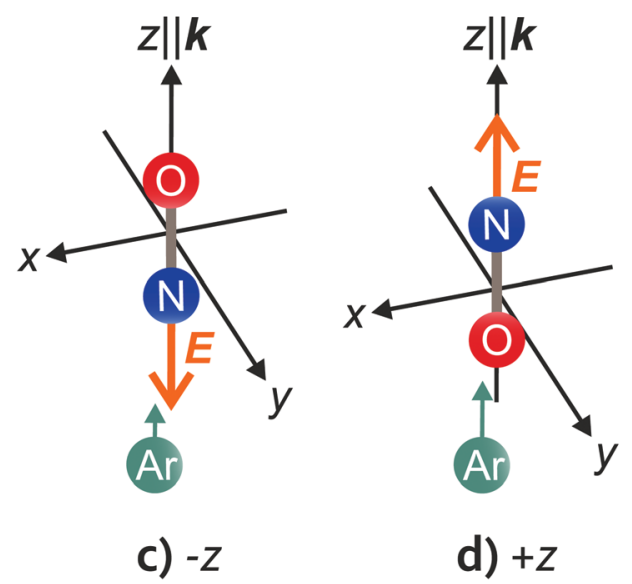

Fig. 1 Schematic representations of the two side-on (top) and the two end-on (bottom) configurations in the scattering frame, in which the $z$-axis is defined parallel to the initial relative velocity vector, $\boldsymbol{k}=\boldsymbol{v}_{\mathrm{Ar}}-\boldsymbol{v}_{\mathrm{NO}}$, and $\boldsymbol{k}$ and $\boldsymbol{k}^{\prime}\left(=\boldsymbol{v}_{\mathrm{Ar}}{ }^{\prime}-\boldsymbol{v}_{\mathrm{NO}}{ }^{\prime}\right.$ ) define the $+x z$ plane. The scattering angle, $\theta$, is indicated in (a). The $+x,-x,-z$, and $+z$ orientations, depicted in (a) $-(d)$, are labelled according to the axis along which the electric field vector, $\boldsymbol{E}$, is directed in each case. Note that the $+x$ and the $-z$ orientations correspond to repulsive collisions of the approaching Ar atom towards the $\mathrm{N}$-side/ $\mathrm{N}$-end, whereas the $-x$ and $+z$ orientations correspond to repulsive collisions towards the $\mathrm{O}$-side/O-end of the $\mathrm{NO}$ molecule.

We define the orientation in the two side-on configurations according to the electric field vector in the scattering frame. In the scattering frame, the initial relative velocity vector, $\boldsymbol{k}$, is parallel to the $z$-axis and the $+x z$ plane is defined by $\boldsymbol{k}$ and $\boldsymbol{k}^{\prime}$, the outgoing relative velocity vector (and the $y$-axis is chosen such that the frame is right-handed). If the electric field points along the $+x$-axis, the orientation is " $+x$ "; if the field points along the $-x$-axis, the orientation is " $-x$ ". The two side-on, as well as the two end-on geometries are depicted schematically in Fig. 1. Note that in the side-on configuration, the $+x$ orientation corresponds to repulsive collisions off the $\mathrm{N}$-side (a), and the $-x$ orientation to repulsive collisions off the O-side of the molecule (b). A closer look at Fig. 1(a) and (b) shows that each of the two side-on geometries actually contains both configurations; if $\boldsymbol{k}^{\prime}$ was lying in the $-x$ hemisphere, the $+x$ orientation in panel (a) would become $-x$ and vice versa for the $-x$ orientation in panel (b).
Therefore, the azimuthal angle of $\boldsymbol{k}^{\prime}$ (in the detector frame) has to be taken into account when analyzing the side-on data. In the end-on configuration, the distribution of $\boldsymbol{k}^{\prime}$ around $\boldsymbol{k}$ is symmetric, and $\mathrm{N}$-end/O-end collisions are defined as " $-z$ " and " $+z$ ”, irrespective of the azimuthal angle of $\boldsymbol{k}^{\prime}$ (Fig. 1(c) and (d)).

A consequence of the asymmetry of the side-on scattering process with respect to $\boldsymbol{k}$ is that the experimental velocity-map ion images contain the scattering distributions of both orientations to either side of $\boldsymbol{k}^{50,51}$ (with $\boldsymbol{k}$ pointing roughly diagonal from the top left to the bottom right corner of the image, dividing it into two parts - this is indicated in the top left image in Fig. 2). Furthermore, due to the variation in detection probability associated with the experimental geometry, the signal intensity is more intense on the lower left side and weaker towards the top right side of the images. The lower left side is where the laboratory velocity is close to zero ("slow" side) and ions in that region have a higher detection probability than ions closer to the top right side that move faster in the laboratory frame ("fast" side). Because most of the intensity in each configuration is located in the lower left portion of the image, we label the images according to the orientation on that side. For the analysis, however, both sides are taken into account.

\section{B. Theoretical and computational methods}

1. Theory. Due to the open-shell nature of the NO, collisions with a rare gas atom take place on two coupled surfaces, labelled $\mathrm{A}^{\prime}$ and $\mathrm{A}^{\prime \prime}$, depending on whether the orbital of the unpaired electron lies within $\left(\mathrm{A}^{\prime}\right)$ the plane defined by the NO and the rare gas atom or perpendicular $\left(\mathrm{A}^{\prime \prime}\right)$ to it. For scattering calculations, it is convenient to work with the sum and difference of the $\mathrm{A}^{\prime}$ and $\mathrm{A}^{\prime \prime}$ potentials, ${ }^{78,79}$ which are defined, respectively, as

$$
V_{\text {sum }}(\gamma, R)=\frac{1}{2}\left[V_{\mathrm{A}^{\prime \prime}}(\gamma, R)+V_{\mathrm{A}^{\prime}}(\gamma, R)\right]=\sum_{\lambda=0}^{\lambda_{\max }} V_{\lambda 0}(R) d_{00}^{\lambda}(\gamma),
$$

and

$$
V_{\mathrm{dif}}(\gamma, R)=\frac{1}{2}\left[V_{\mathrm{A}^{\prime \prime}}(\gamma, R)-V_{\mathrm{A}^{\prime}}(\gamma, R)\right]=\sum_{\lambda=2}^{\lambda_{\max }} V_{\lambda 2}(R) d_{20}^{\lambda}(\gamma) .
$$

Here, $R$ is the distance between the Ar atom and the center of mass of the NO and $\gamma$ is the angle between $R$ and the NO bondaxis, $r$. The $V_{\lambda m}(R)$ are the radially dependent expansion coefficients and the $d_{m n}^{\lambda}(\gamma)$ are reduced Wigner rotation matrix elements. ${ }^{80}$ When the orbital angular momentum of the molecule is strongly coupled to the intermolecular axis, i.e., in a pure Hund's case (a), the scattering problem for spin-orbit conserving transitions can be solved exactly using the sum potential only. However, for spin-orbit changing transitions (and deviations from a pure Hund's case (a)), the off-diagonal matrix coupling elements, which are encoded in the difference potential, are required to obtain accurate scattering amplitudes. ${ }^{78,79,81}$

As shown in our previous work, ${ }^{50,51}$ the orientation dependent differential cross section (DCS) can be expressed in terms of the molecular bond-axis dependent polarization moments, or $r$-PDDCSs. The $r$-PDDCSs themselves are a function of the relevant scattering amplitudes, as defined in the ESI. $\dagger$ For a 

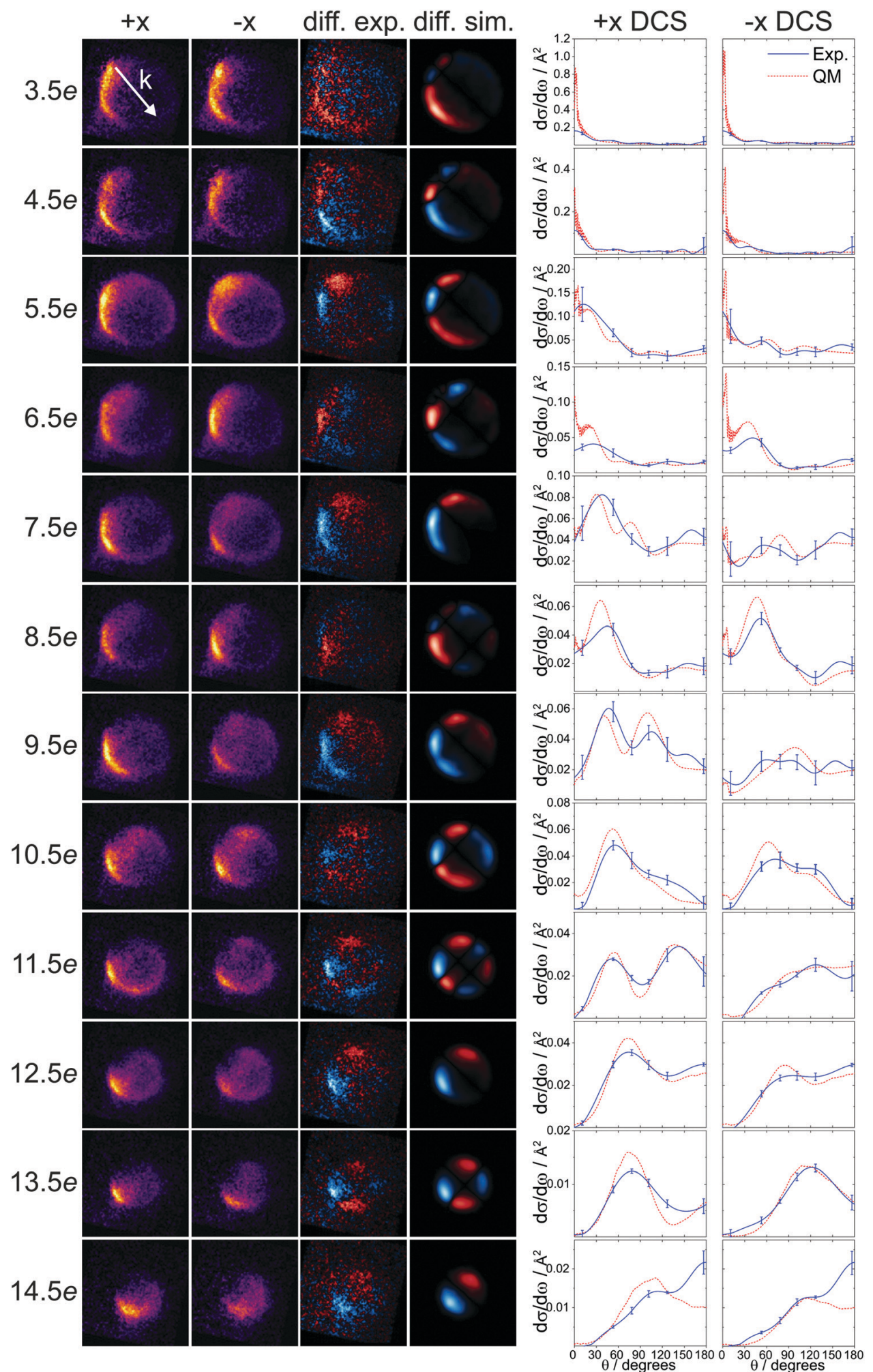

Fig. 2 Experimental velocity-map ion images and DCSs compared with QM results $(\Delta \Omega=1$ ). Experimental $+x$ and $-x$ ion images (columns 1 and 2 ), experimental and QM simulated difference images (columns 3 and 4), and differential cross sections for the $+x$ and $-x$ orientations (columns 5 and 6 ) are shown for $j^{\prime}=3.5 e-14.5 e$ (top to bottom). The experimental DCSs (blue lines), with error bars corresponding to one standard deviation, are compared to the QM calculated DCSs (red dashed lines). The QM DCSs are averaged over the spread of the experimental collision energy. The relative velocity vector, $\boldsymbol{k}=\boldsymbol{v}_{\mathrm{Ar}}-\boldsymbol{v}_{\mathrm{NO}}$, is indicated by the white arrow in the top left image. 
specific transition from an initial state $|j=1 / 2| m_{\mathrm{E}}|\Omega E\rangle$ to a final state $\left|j^{\prime} \Omega^{\prime} E^{\prime}\right\rangle$, the DCS corresponds to:

$$
\begin{aligned}
{\left[\mathrm{d} \sigma\left(j\left|m_{E}\right| \Omega \hat{E} \rightarrow j^{\prime} \Omega^{\prime} \varepsilon^{\prime}\right)\right]_{\theta_{E}}^{\phi_{E}}=} & \frac{\sigma_{\text {iso }}}{2 \pi}\left\{R_{0}^{(0)}(\theta)-|\alpha \beta|\left[R_{0}^{(1)}(\theta) \cos \theta_{E}\right.\right. \\
& \left.\left.-\sqrt{2} R_{1}^{(1)}(\theta) \sin \theta_{E} \cos \phi_{E}\right]\right\},
\end{aligned}
$$

where $\sigma_{\text {iso }}$ is the isotropic integral cross section (ICS) in the presence of a field, $\alpha$ and $\beta$ are the field-dependent mixing parameters introduced in eqn (1), and the $R_{q}^{(k)}(\theta)$ are the $r$-PDDCSs. The orientation of the electric field is specified by the polar $\left(\theta_{E}\right)$ and azimuthal $\left(\phi_{E}\right)$ angles of $\boldsymbol{E}$ in the scattering frame. For the side-on orientations, $\theta_{E}=90^{\circ}$ and $\phi_{E}=0^{\circ}(+x)$ or $\phi_{E}=180^{\circ}(-x)$ (see Fig. 1). The end-on orientations are independent of the $\phi_{E}$ angle, and $\theta_{E}=0^{\circ}(+z)$ or $\theta_{E}=180^{\circ}(-z)$. By substituting the values for these angles into eqn (4), it can be seen that for the side-on orientation, the second term containing the $R_{0}^{(1)}(\theta)$ moment goes to zero, while for the end-on orientation, the third term containing the $R_{1}^{(1)}(\theta)$ moment goes to zero. Therefore, for $j=1 / 2$, in which case only the first-order orientation moments exist, $R_{0}^{(1)}(\theta)$ and $R_{1}^{(1)}(\theta)$ quantify orientation along the $z$ - and $x$-axes, respectively, and $R_{0}^{(0)}(\theta)$ represents the isotropic moment. A thorough discussion of the formalism for oriented DCSs is given in the ESI. $\dagger$

In previous work ${ }^{82,83}$ it was demonstrated that it is possible to define a quantum generalized deflection function (GDF), a quantum analog to the classical joint probability distribution of the scattering angle $\theta$ and the total angular momentum $J$, which can be written as:

$$
\begin{aligned}
Q(\theta, J)= & \frac{\sin \theta}{2 j+1} \sum_{J_{1}} \sum_{J_{2}} \sum_{m, m^{\prime}} \frac{\delta_{J_{1}, J}+\delta_{J_{2}, J}}{2} \\
& \times F_{m^{\prime} \varepsilon^{\prime}, m \varepsilon}^{J_{1}}(\theta)\left[F_{m^{\prime} \varepsilon^{\prime}, m \varepsilon}^{J_{2}}(\theta)\right]^{*},
\end{aligned}
$$

where the initial and final $j$ and $\Omega$ quantum numbers have been omitted for simplicity. The scattering amplitude $F_{m^{\prime} \varepsilon^{\prime} m \varepsilon}^{J}(\theta)$ is defined as:

$$
F_{m^{\prime} \varepsilon^{\prime} m \varepsilon}^{J}(\theta)=\frac{1}{2 i k_{\mathrm{in}}}(2 J+1) d_{m^{\prime} m}^{J}(\theta) S_{j^{\prime} m^{\prime} \Omega^{\prime} \varepsilon^{\prime}, j m \Omega \varepsilon}^{J},
$$

with $k_{\text {in }}$ denoting the initial relative wave vector, $d_{m^{\prime} m}^{J}(\theta)$ the reduced rotation matrix element, and $S_{j^{\prime} m^{\prime} \Omega^{\prime} \varepsilon^{\prime}, j m \Omega \varepsilon}^{J}$ the scattering matrix element for the specific transition.

In contrast to its classical counterpart, the QM GDF also accounts for coherences between $J$-partial waves, which enables the observation of interference patterns and the disentanglement of the $J$-partial waves that contribute to constructive and destructive interference. The QM GDF can therefore provide valuable insights into the scattering mechanism. ${ }^{82}$

As shown in the ESI $\dagger$ (see also ref. 83), for electric field orientation of the $j=1 / 2$ initial state, the orientation dependent QM GDF can be expressed as:

$$
\begin{aligned}
{[Q(\theta, J)]_{\theta_{E}}^{\phi_{E}}=} & \sin \theta \frac{\sigma_{\text {iso }}}{2 \pi}\left\{Q_{0}^{(0)}(\theta, J)-|\alpha \beta|\left[Q_{0}^{(1)}(\theta, J) \cos \theta_{E}\right.\right. \\
& \left.\left.-\sqrt{2} Q_{1}^{(1)}(\theta, J) \sin \theta_{E} \cos \phi_{E}\right]\right\},
\end{aligned}
$$

where the polarization dependent QM GDFs, $Q_{q}^{(k)}(\theta, J)$, are a function of both $\theta$ and $J$, and are defined similarly to the $R_{q}^{(k)}(\theta)$ moments in eqn (4). Further details, including the derivation of the QM GDF, are presented in the ESI. $\dagger$

Integration of eqn (7) over $\theta$ yields the $J$-partial cross section,

$$
[\tilde{\sigma}(J)]_{\theta_{E}}^{\phi_{E}}=\int_{0}^{\pi}[Q(\theta, J)]_{\theta_{E}}^{\phi_{E}} \mathrm{~d} \theta=\frac{1}{2{k_{\mathrm{in}}}^{2}}(2 J+1)[\tilde{P}(J)]_{\theta_{E}}^{\phi_{E}},
$$

with $[\tilde{P}(J)]_{\theta_{E}}^{\phi_{E}}$ denoting the orientation dependent collision probability, or opacity function.

2. Computational details. The close-coupled quantum mechanical (CC-QM) calculations were carried out with the Hibridon suite of codes, ${ }^{84}$ employing the potential energy surfaces by Alexander. ${ }^{52,53}$ Rotational states up to $j^{\prime}=20.5$, both spin-orbit manifolds $(\Omega=1 / 2,3 / 2)$, and both $\Lambda$-doublet levels were included in the scattering calculation. 190 partial waves $(J=l+j, \ldots,|l-j|)$ were used to ensure convergence. The scattering amplitudes for the individual $\Lambda$-doublet resolved transitions, obtained in the Hibridon calculation, were combined to determine the $r$-PDDCSs (see ESI $\dagger$ ), which were then used to calculate the bond-axis oriented differential cross sections according to eqn (4).

To allow comparison with the experiment, the experimental velocity distribution was accounted for by averaging the DCSs calculated at seven different collision energies in the range between 590 and $710 \mathrm{~cm}^{-1}$, each weighted to a Gaussian distribution function with a mean collision energy of $651 \mathrm{~cm}^{-1}$ and a FWHM of $35 \mathrm{~cm}^{-1}$. For the theoretical analysis, the DCSs for the two side-on $( \pm x)$ and the two end-on $( \pm z)$ orientations were also calculated in the limit of infinite field (and at a fixed $E_{\text {coll }}=651 \mathrm{~cm}^{-1}$ ), with calculations run separately on the $\mathrm{A}^{\prime}$ and $\mathrm{A}^{\prime \prime}$ PESs, as well as the sum and the full PESs. For the calculations on the $\mathrm{A}^{\prime}, \mathrm{A}^{\prime \prime}$, and sum PESs, which effectively describe a closed-shell system, only the physically meaningful spin-orbit conserving transitions were considered. On the full potential, the infinite field DCSs were computed for both spin-orbit manifolds.

\section{Results}

\section{A. Velocity-map ion images and differential cross sections}

The experimental velocity-map ion images for the spin-orbit excited transitions following inelastic collisions of $\mathrm{NO}$ with $\mathrm{Ar}$ are presented in Fig. 2. The images for the $+x$ and the $-x$ orientations are shown in the first and second column, and the difference images, obtained by subtracting the $-x$ image from the $+x$ image, are shown in the third column. These are compared to the simulated difference images, using the QM polarization moments, in the fourth column. The intensity scale is identical in the $+x$ and $-x$ images for each rotational state; the intensities are therefore directly comparable.

Overall, there is good agreement between the experimental and QM simulated data, indicating the high accuracy of the PESs used and a solid understanding of the experimental parameters. As the rotational excitation of the NO increases (from $3.5 e$ to $14.5 e$, top to bottom), the outgoing kinetic energy 
decreases, resulting in smaller Newton spheres. It can also be seen that, in general, the distributions become more and more backward scattered as a function of increasing rotational excitation, reflecting the requirement for more impulsive encounters (and consequently more backward scattered trajectories) to access higher lying rotational levels.

Similar to our previous results for the spin-orbit conserving manifold ${ }^{50,51}$ and end-on oriented collisions, ${ }^{42,72}$ an alternation in overall scattering intensity between the $+x$ and $-x$ configurations is observed as a function of $\Delta j$. We have rationalized this alternation in steric preference by quantum interference, in which the sign of the phase shift between interfering scattering amplitudes changes as a function of $\Delta j \cdot{ }^{50,51}$ In the current data, the alternation is most clearly seen in the difference images, where blue on the slow side of the image corresponds to a preference for the $+x$ orientation (positive intensity), and red indicates a preference for the $-x$ orientation (negative intensity). On the fast side of the images, the colors (and intensities) are inverted, as on this side, the $+x$ orientation is subtracted from the $-x$ orientation. For odd $\Delta j$ transitions, the $+x$ orientation is preferred, especially in the forward scattered direction. For even $\Delta j$ transitions up to $\Delta j=10$, the $-x$ orientation, although to a smaller degree, dominates. For transitions with $\Delta j \geq 12$, the $+x$ orientation is preferred, irrespective of whether $\Delta j$ is odd or even.

The differential cross sections extracted from the experimental images and the corresponding QM calculated DCSs for the $+x$ and $-x$ orientations are shown on the right-hand side of Fig. 2 . The match between experiment and calculation is good; for $j^{\prime}=$ $11.5 e-13.5 e$, the agreement is excellent, while for some of the other states, especially the intermediate ones around $j^{\prime}=6.5 e-9.5 e$, the agreement is somewhat poorer. We attribute the reduced quality of the fitted data for these particular states to the fact that the main features overlap with the region where the laboratory velocity is close to zero and the detection efficiency is highest. ${ }^{49,72}$ As a consequence, any small inaccuracies in the instrument function will lead to noticeable deviations from the true DCS.

Compared to the spin-orbit conserving DCSs, the DCSs in the current data set show intensity over the entire range of scattering angles, are generally more backward scattered, and have features that are broader and less sharp. The dominance of the $+x$ orientation for odd $\Delta j$ transitions is observed clearly, particularly in the forward scattered direction $\left(\theta \leq 90^{\circ}\right)$ for the transitions between $j^{\prime}=5.5 e-11.5 e$. For even and higher $\Delta j$ transitions, on the other hand, the DCSs for the two side-on orientations are more similar, both in shape and magnitude.

The ion images and DCSs measured for the spin-orbit excited state in the end-on orientation have exhibited similar structures, and followed the same odd-even alternation, as the current experimental data, although the difference in intensity between the $+z$ and $-z$ geometries were somewhat more pronounced than between the two side-on orientations. ${ }^{72}$

\section{B. Integral steric asymmetries (ISA)}

The integral steric asymmetry quantifies the extent to which one orientation is preferred over the other. For the $x$-axis configuration, the steric asymmetry, $S_{x}$, is calculated from the integrated differential cross sections (eqn (4)) for the $+x$ and $-x$ orientations, $\sigma_{ \pm x}$ :

$$
S_{x}=\frac{\sigma_{+x}-\sigma_{-x}}{\sigma_{+x}+\sigma_{-x}} \times 100 .
$$

The ISA for $z$-axis orientation, $S_{z}$, is defined analogously as:

$$
S_{z}=\frac{\sigma_{-z}-\sigma_{+z}}{\sigma_{+z}+\sigma_{-z}} \times 100 .
$$

As will be discussed below, all collisions in the spin-orbit changing manifold are nearside, such that the $+x /-z$ orientation corresponds to repulsive scattering off the $\mathrm{N}$-side/ $\mathrm{N}$-end, while the $-x /+z$ configuration corresponds to repulsive scattering off the O-side/O-end. A positive ISA therefore indicates a preference for repulsive scattering off the $\mathrm{N}$-side/ $\mathrm{N}$-end, and a negative ISA indicates a preference for repulsive scattering off the O-side/O-end.

Fig. 3 shows the experimental ISA (blue) along with the collision energy averaged QM ISA (red) for the $x$-axis orientation
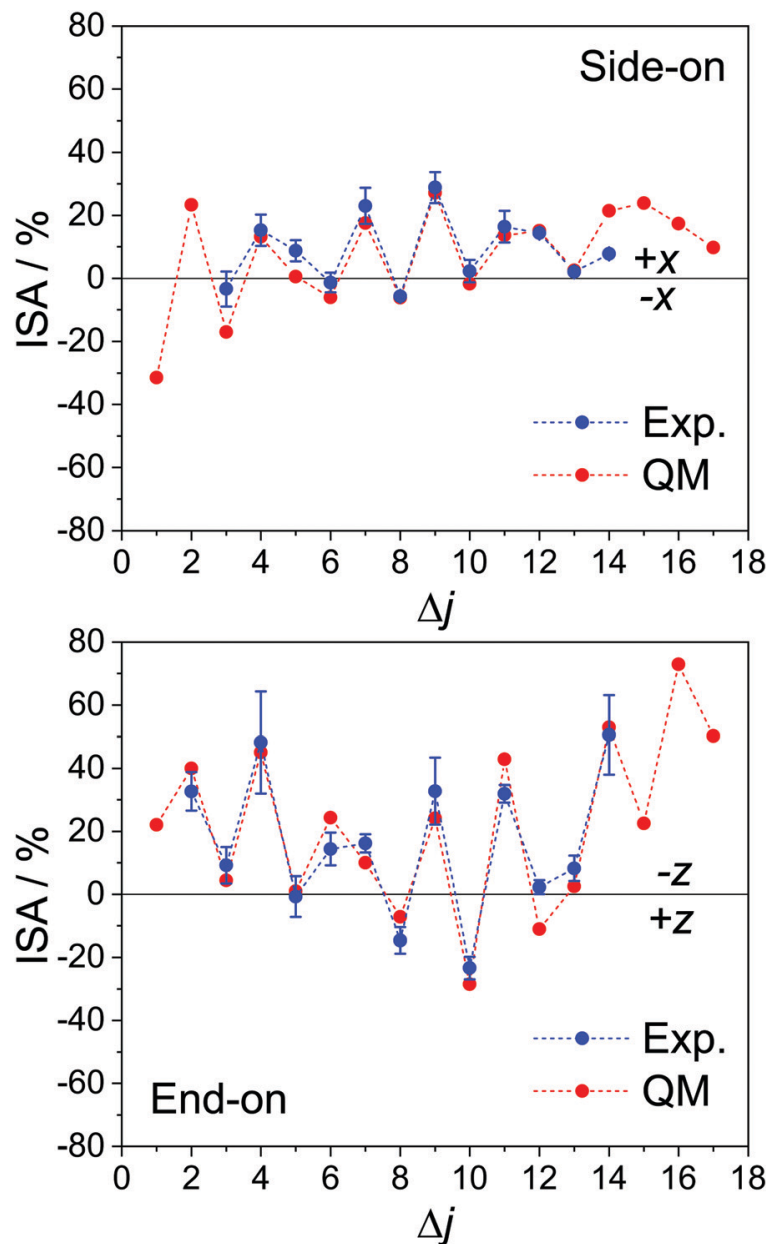

Fig. 3 Experimental and QM calculated integral steric asymmetries for the spin-orbit excited manifold $(\Delta \Omega=1)$. The experimentally measured ISA is shown in blue, with error bars representing one standard deviation; the corresponding QM data, which have been averaged over the experimental collision energy distribution, are shown in red. The top panel is for the $x$-axis configuration, probed in the current experiment, and the bottom panel is for the $z$-axis orientation (with the experimental data taken from ref. 85). Both sets of data are for the final e $\Lambda$-doublet states. 
from the current experiment (top panel) and, for comparison, the $z$-axis orientation from previous work in our group. ${ }^{85}$

As with the DCSs, there is good agreement between the experimental and QM calculated ISAs, and the alternation in preference between odd and even $\Delta j$ is evident. However, in both the side-on and the end-on configurations, the $\mathrm{N}$-side/ $\mathrm{N}$-end preference is significantly larger in magnitude than the preference for the O-side/O-end. In fact, for $\Delta j=6,8,10$ in the $x$-axis configuration, the ISA is close to zero. The observed trend points to an overall preference for collisions off the $\mathrm{N}$-side/ $\mathrm{N}$-end of the molecule and contrasts with the steric asymmetries we have measured for the corresponding spin-orbit conserving transitions, for which the ISAs for adjacent $\Delta j$ transitions are similar in magnitude ${ }^{42,51}$ (see Fig. S1 in the ESI $\dagger$ ). Note that for $\Delta j=5$ in the side-on orientation, the ISA is also approximately zero, even though the main feature in the forward scattered direction is larger in the $+x$ DCS than in the $-x$ DCS (Fig. 2). The small ISA is partly due to the fact that upon integration, the intensity is weighted by $\sin \theta$ (to account for the solid angle), and partly because the $-x$ DCS is consistently larger in the backward scattered region $\left(\theta \geq 90^{\circ}\right)$.

\section{Calculated DCSs and QM deflection functions at infinite field}

Experimentally, the extent to which the NO molecules can be oriented is limited in part by the electric field strength; in order to eliminate any effects due to incomplete initial orientation of the molecules, we have calculated the DCSs at infinite field, at which the mixing of the initial $e$ and $f \Lambda$-doublets is complete (i.e., both states contribute equally to the initial superposition state, as $|\alpha|=|\beta|=1)$. Note that even at infinite field, the initial orientation of the NO molecules is still defined by a relatively broad cosine probability distribution, in which the most probable orientation is the one in which the $\mathrm{NO}$ axis $(\mathrm{N} \rightarrow \mathrm{O})$ is antiparallel to the electric field vector, but other orientations, weighted according to the cosine distribution, contribute as well (see, for example, Fig. 3 in ref. 42). Fig. 4 presents the infinite field DCSs for the $+x$ (red), $-x$ (blue dashed), $+z$ (purple dashed) and $-z$ (green) orientations, along with the maximized DCSs (gray shaded area), for $\Delta j=4 e-13 e$. The maximized DCS corresponds to the orientation (as defined by $\theta_{E} / \phi_{E}$ ) that maximizes eqn (4) at a given scattering angle. Note that at $\theta=0^{\circ} / 180^{\circ}$, the DCSs for the $-x$ and $+x$ configurations are identical, since at those angles the orientation is along the $\pm z$-axis.

For the odd $\Delta j$ transitions, the trends are similar to the ones for the spin-orbit conserving manifold, ${ }^{50,51}$ with the $+x$ and the $-z$ orientations (collisions off the $\mathrm{N}$-side/ $\mathrm{N}$-end) dominating in the side-on and the end-on configuration, respectively. In addition, the side-on DCSs are close to the maximized DCSs in the forward scattered region, while the end-on DCSs more closely match the maximized DCSs in the backward scattered region. ${ }^{50,51}$

However, the even $\Delta j$ transitions, shown in the top two rows of Fig. 4, follow a different pattern. Most strikingly observed for $\Delta j=6,8,10$ is a distinct peak in the very forward scattered direction that is maximized in the O-end $(+z)$ and minimized in
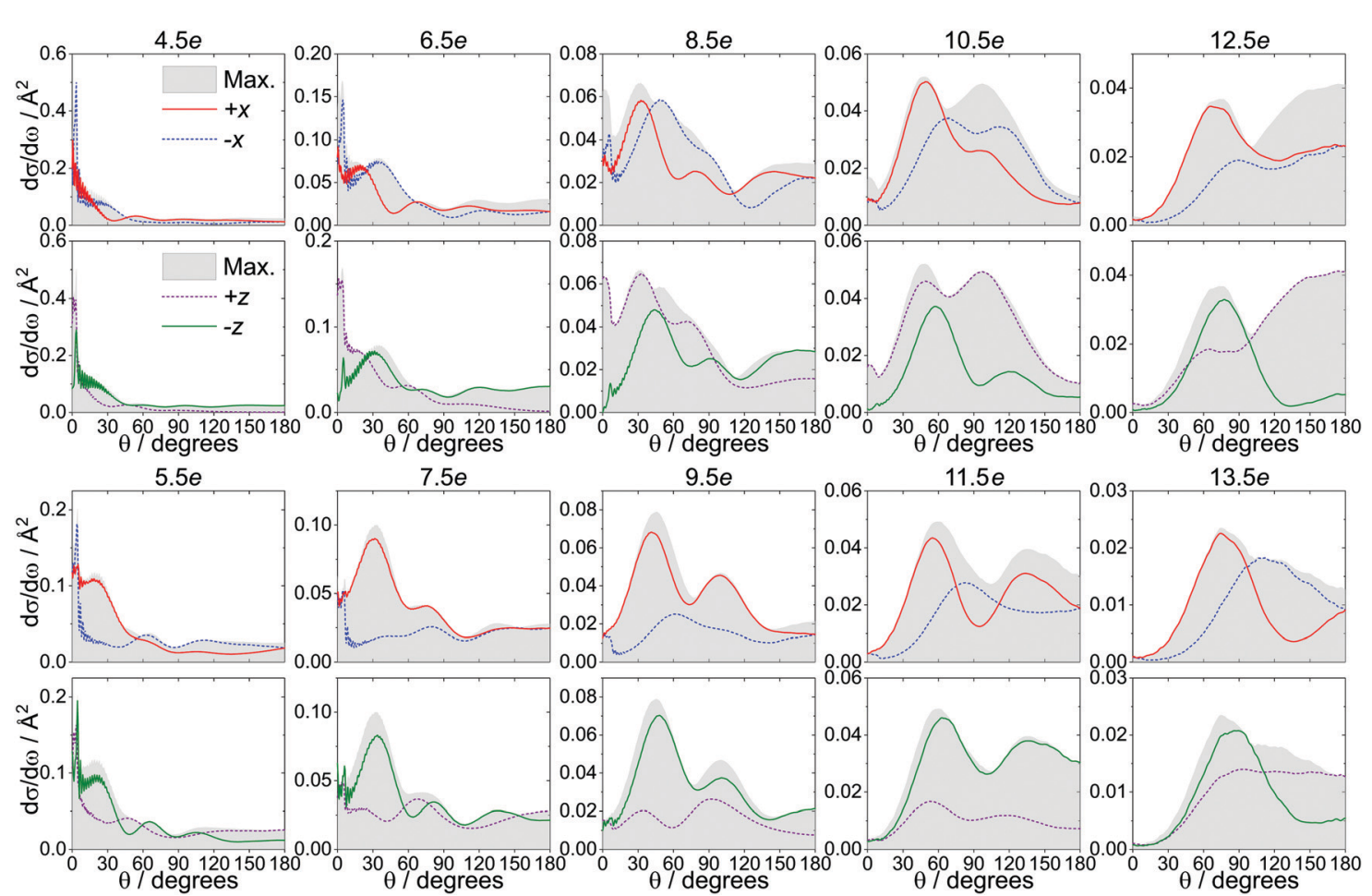

Fig. 4 Comparison of the spin-orbit changing, infinite field DCSs for the side-on (red: $+x$, blue: $-x$ ) and end-on (purple: $+z$, green: $-z$ ) configurations with the maximized DCSs (gray shaded areas). The even transitions between $\Delta j=4-12$ are shown in the two upper rows, and the odd transitions between $\Delta j=5-13$ are shown in the two lower rows. 
the $\mathrm{N}$-end $(-z)$ orientation, with the two side-on orientations representing intermediate cases. At larger values of $\theta$, the magnitude of the $+x$ DCS for $\Delta j \leq 8$ is comparable to that of the $-x$ DCS (which is expected to dominate for the even $\Delta j$ ) and becomes more dominant for $\Delta j \geq 10$, where it closely matches the maximized DCS in the forward scattered region. This significantly diminishes the steric preference for the $-x$ orientation for these even $\Delta j$ states, in agreement with the increased importance of $\mathrm{N}$-side/ $\mathrm{N}$-end collisions observed in the ISA (Fig. 3).

The $J$-partial cross sections, shown in Fig. S3 in the ESI, $\dagger$ corroborate the trends observed in the DCSs in Fig. 4; for the odd $\Delta j$ transitions, the preference for $+x$ and $-z$ is clear, while for the even $\Delta j$ transitions, the two side-on and the two end-on orientations are more similar. Differences are in general more evident at higher impact parameters, which are correlated with smaller scattering angles.

Fig. 5 shows the QM generalized deflection functions (divided by $\sin \theta$ ) for the final $\left|j^{\prime}=8.5, \Omega=3 / 2 e\right\rangle$ and $\left|j^{\prime}=9.5, \Omega^{\prime}=3 / 2 e\right\rangle$ states. As described in Section II B 1. and in ref. 82 and 83, the QM deflection function represents the joint quasi-probability of the scattering angle and total angular momentum. It exposes the range of total angular momenta that contribute to each of the maxima in the DCS. By summing the contributions of all $J$ s (integration or sum along the vertical axis), the DCS is recovered, and similarly, by integration over the scattering angle, the $J$-partial cross section is obtained.

The QM $J-\theta$ correlations in Fig. 5 are indicative of direct collisions: high $J$ lead to low scattering angles, and vice versa. Although the GDFs extend over the entire range of scattering angles, the $J-\theta$ mutual dependence is diagonal and continuous, which rules out the presence of resonances or long-lived complexes. The negative slope indicates that all collisions are nearside. Moreover, the interval of partial wave coherences is small, and hence interference between different groups of partial waves is largely irrelevant except at the extreme forward angles.

In spite of these common features, the specific structure of the GDFs for the different orientations, and the even and odd $\Delta j$ transitions, differ substantially, suggesting distinct scattering mechanisms. In both states shown in Fig. 5 , the $\mathrm{N}$-side $(+x$, bottom left) and $\mathrm{N}$-end ( $-z$, top right) and the O-side $(-x$, top left) and $\mathrm{O}$-end $(+z$, bottom right) deflection functions exhibit similar features. For $\Delta j=8$, the O-side/O-end orientations are slightly dominant over the $\mathrm{N}$-side/ $\mathrm{N}$-end orientations, whereas for $\Delta j=9$, the $\mathrm{N}$-side/ $\mathrm{N}$-end orientations are significantly more intense than the O-side/O-end orientations. This again mirrors the overall dominance of $\mathrm{N}$-side/ $\mathrm{N}$-end collisions over $\mathrm{O}$-side/ O-end collisions in the spin-orbit excited manifold, as observed in Fig. 3.

The prominent forward peak close to $\theta \sim 0^{\circ}$ is strongly correlated with a small range of high impact parameters $(J \sim 80-90)$. In the $8.5 e$ final state, the feature is particularly intense in the $+z$ orientation, but is only weak in the $+x$ and essentially absent in the $-z$ orientation. In the 9.5e state, the peak has similar intensity in all four configurations.
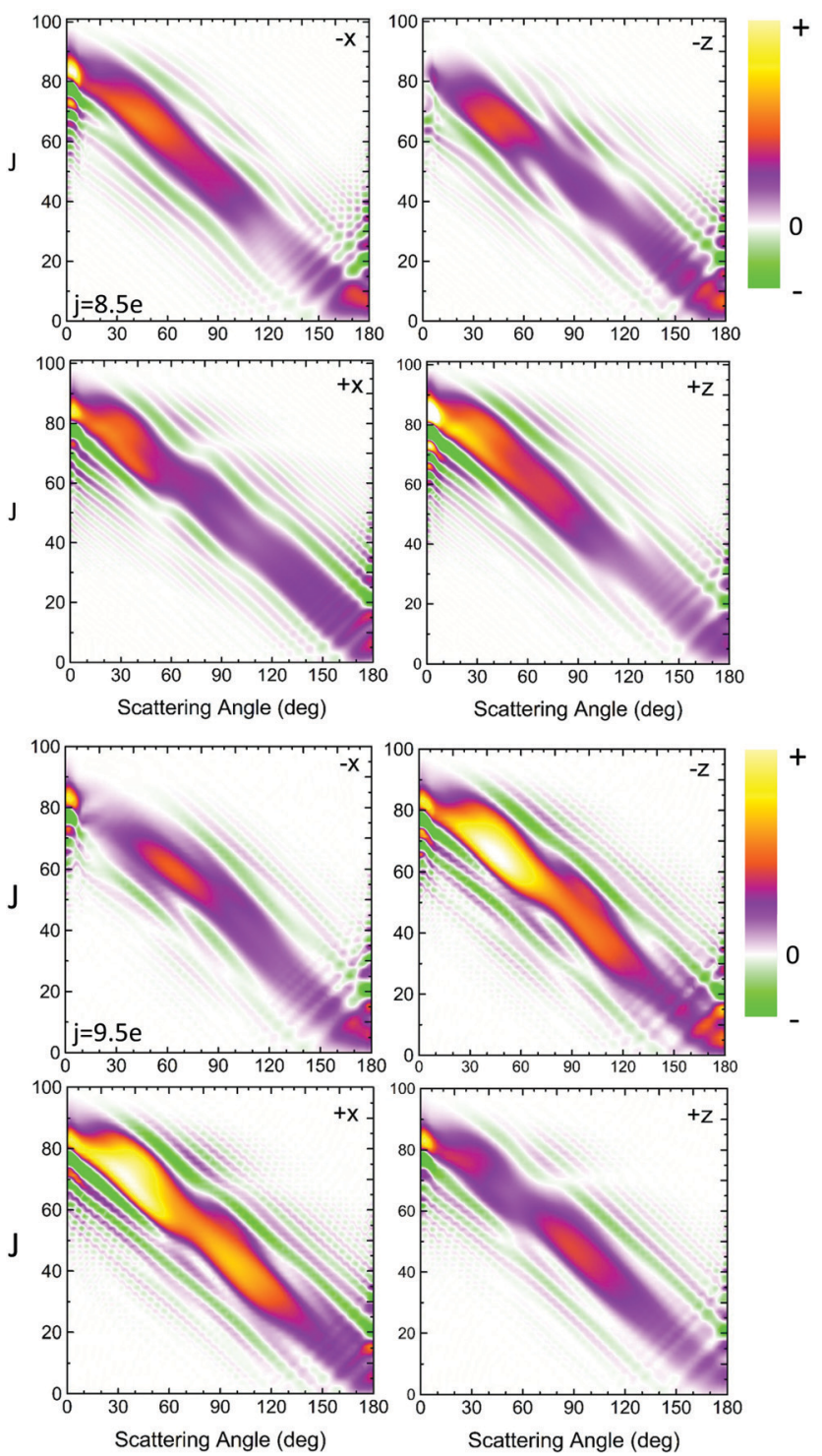

Fig. 5 QM generalized deflection functions for the $j^{\prime}=8.5 e$ (top) and $j^{\prime}=9.5 e$ (bottom) final rotational states in the $\Delta \Omega=1$ manifold. In both states, the $+x$ and $-z$ ( $\mathrm{N}$-side/ $\mathrm{N}$-end) and the $-x$ and $+z(\mathrm{O}$-side/O-end) orientations show similar features, with $+x /-z$ clearly dominating in the odd $\Delta j=9$ state. The forward peak $\left(\theta \sim 0^{\circ}\right)$ is correlated with high impact parameters $(J>80)$ and appears especially pronounced in the $+z$ configuration for $j^{\prime}=8.5 e$. The generalized deflection functions (eqn (7)) have been divided by $\sin \theta$ to highlight forward and backward scattering.

\section{Discussion}

\section{A. $\mathbf{N}$-side/N-end preference}

The observed trends in the calculated DCSs and QM deflection functions for the even and odd $\Delta j$ transitions indicate that the scattering dynamics into the spin-orbit excited manifold are fundamentally different to the dynamics into the spin-orbit conserving manifold. In order to unravel these differences, we turn to the potential energy surfaces that govern the scattering process in each case. As already mentioned, collisions of the NO molecule with a rare gas atom occur on the coupled $\mathrm{A}^{\prime}$ and $\mathrm{A}^{\prime \prime}$ potentials. In a quasi-classical approach, Zhang and Stolte 
recently used an expression which explicitly takes into account the position of the unpaired electron with respect to the triatomic plane: ${ }^{86}$

$$
\begin{aligned}
V(R, \gamma, \chi)= & \frac{1}{2}\left[V_{\mathrm{A}^{\prime \prime}}(R, \gamma)+V_{\mathrm{A}^{\prime}}(R, \gamma)\right] \\
& -\frac{1}{2}\left[V_{\mathrm{A}^{\prime \prime}}(R, \gamma)-V_{\mathrm{A}^{\prime}}(R, \gamma)\right] \cos (2 \chi) .
\end{aligned}
$$

The first and second term (excluding the $\cos (2 \chi)$ dependence) are the sum and difference potentials introduced in eqn (2) and (3), and $\chi$ is the angle of the unpaired electron with respect to the triatomic plane.

For spin-orbit conserving transitions, it is favorable if the interaction between the unpaired electron and the approaching Ar atom is minimal. In order to effect a spin-orbit excitation, on the other hand, the Ar atom necessarily needs to interact with the unpaired electron. In the two limiting cases of the unpaired electron lying within $\left(\chi=0^{\circ}\right.$, preferred for spin-orbit changing transitions) or perpendicular $\left(\chi=90^{\circ}\right.$, preferred for spin-orbit conserving transitions) to the plane defined by the three atoms, eqn (11) gives:

$$
\begin{gathered}
V\left(R, \gamma, \chi=0^{\circ}\right)=V_{\mathrm{A}^{\prime}}(R, \gamma) \\
V\left(R, \gamma, \chi=90^{\circ}\right)=V_{\mathrm{A}^{\prime \prime}}(R, \gamma)
\end{gathered}
$$

Consequently, we expect the spin-orbit changing manifold to be predominantly governed by the $\mathrm{A}^{\prime}$ potential, and the spinorbit conserving transitions by the $\mathrm{A}^{\prime \prime}$ potential.

The $\mathrm{A}^{\prime}, \mathrm{A}^{\prime \prime}$, and sum PESs are plotted in Fig. 6. The red lines represent the repulsive core, while the blue lines indicate the attractive parts of the potential. As seen in the figure, the $\mathrm{A}^{\prime}$ PES is wider on the $\mathrm{N}$-side and features a concave repulsive region on the side of the molecule. The extended width on the N-side indicates the location of the unpaired electron and collisions towards this end of the molecule are expected to be more likely to lead to spin-orbit excitation than collisions towards the O-side/O-end of the molecule. The $\mathrm{A}^{\prime \prime}$ and sum PESs, in contrast, are convex. The widest part of the $\mathrm{A}^{\prime \prime}$ PES is close to the center of the molecular axis and interaction with the unpaired electron, which in this case lies out of plane, is less efficient.
These considerations imply two things: firstly, the $\mathrm{A}^{\prime}$ potential, as indicated in eqn (12), plays a crucial role for spin-orbit changing transitions (and less so for spin-orbit conserving transitions). Secondly, the overall shift of the ISA towards an $\mathrm{N}$-side/ $\mathrm{N}$-end preference is a direct consequence of the location of the unpaired electron. For odd $\Delta j$ transitions, in which $\mathrm{N}$-side/ $\mathrm{N}$-end collisions are also favored based on quantum interference, the trends are similar to the ones in the spin-orbit conserving manifold. For the even $\Delta j$ transitions, in which quantum interference is constructive for $\mathrm{O}$-side/O-end collisions, the required interaction with the unpaired electron renders trajectories towards the $\mathrm{N}$-side/N-end more important than in the spin-orbit conserving manifold. This results in comparable contributions from $\mathrm{N}$-side/N-end and $\mathrm{O}$-side/O-end collisions, and consequently a relatively small steric asymmetry, for even $\Delta j$ transitions in the spin-orbit excited manifold.

\section{B. The prominent forward scattered peak}

In addition to the overall $\mathrm{N}$-side/ $\mathrm{N}$-end preference in the spinorbit changing manifold, a second intriguing feature in our calculations is the prominent forward peak observed for even $\Delta j$ transitions, particularly the states between $\Delta j=6-10$, in the O-end orientation.

Fig. 7 examines the contributions of the $\mathrm{A}^{\prime}$ (first column) and $\mathrm{A}^{\prime \prime}$ (second column) potentials to the total DCSs for the spin-orbit conserving ( $\Delta \Omega=0$, third column) and changing ( $\Delta \Omega=1$, fourth column) transitions for $j^{\prime}=8.5 e$. This state was chosen because of the prominence of its forward scattered peak, but the results for other intermediate $\Delta j$ transitions are overall very similar. The DCSs for the side-on and the end-on orientations are shown at the top and bottom of Fig. 7, respectively. The DCSs obtained on the $\mathrm{A}^{\prime}$ potential exhibit a strong forward scattered peak in all orientations except the $\mathrm{N}$-end $(-z)$ configuration, whereas on the $\mathrm{A}^{\prime \prime}$ potential, only a small sharp peak within a broader feature in the forward direction is observed. The spin-orbit conserving transitions calculated on the full PES resemble the DCSs calculated on the $\mathrm{A}^{\prime \prime}$ PES, confirming that these transitions occur to a large extent on the $\mathrm{A}^{\prime \prime}$ potential. The spin-orbit changing transitions appear to
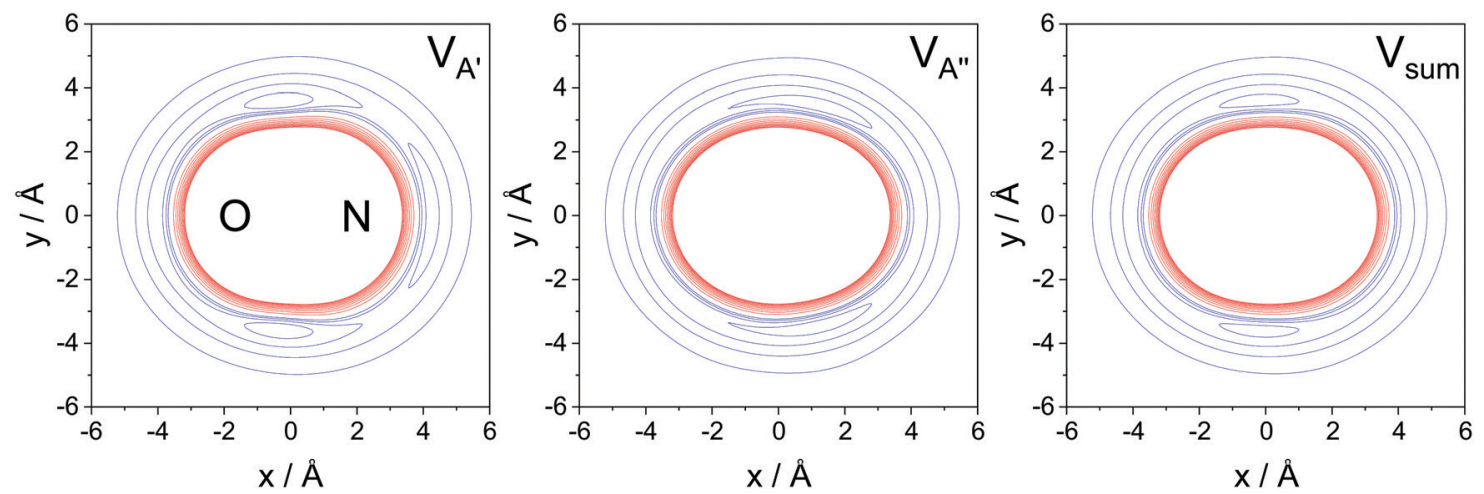

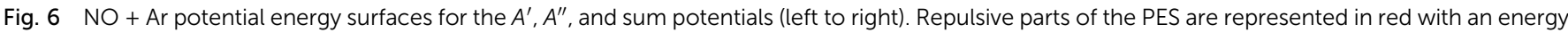

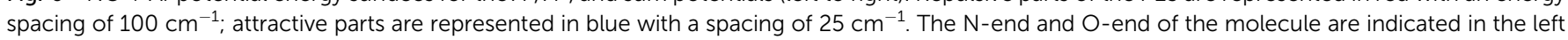
panel. 

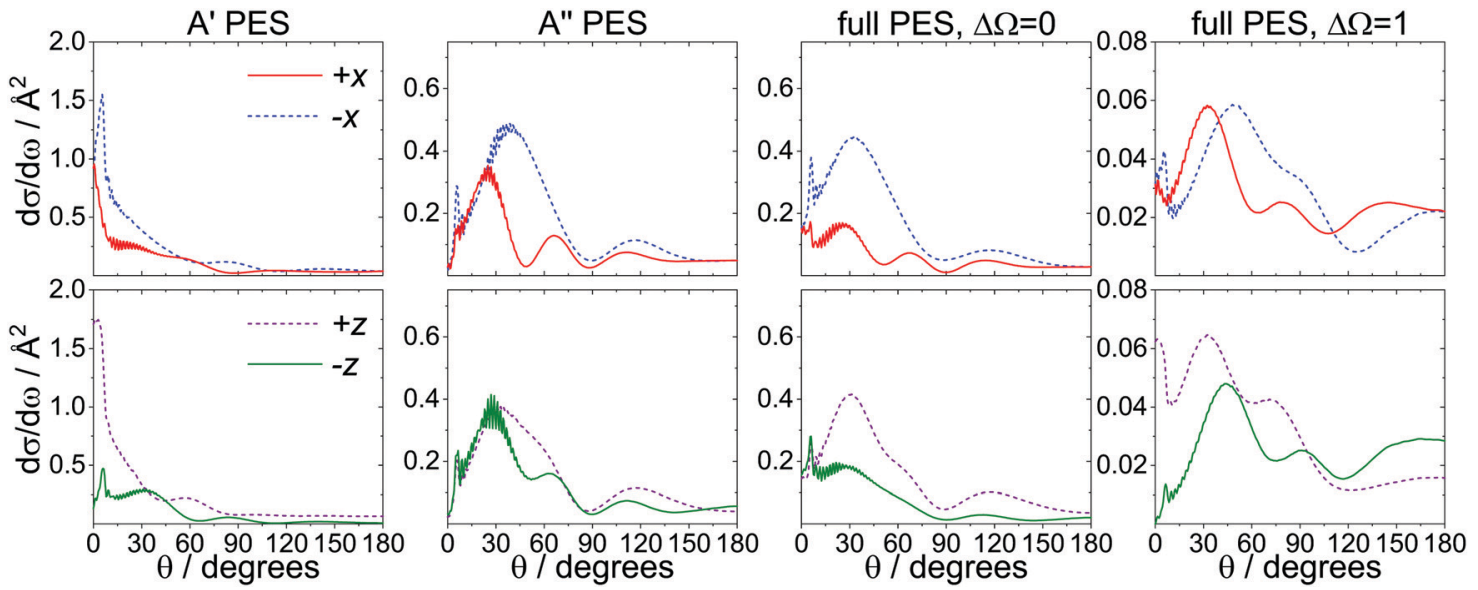

Fig. 7 Infinite field QM DCSs for $\Delta j=8$, calculated on the $\mathrm{A}^{\prime}, \mathrm{A}^{\prime \prime}$, and full potentials, in the $\pm x$ (top) and the $\pm z$ (bottom) orientations. The calculations run on the $A^{\prime}$ and $A^{\prime \prime}$ PESs (first and second columns) correspond to spin-orbit conserving transitions. The third and fourth columns show the DCSs calculated on the full potential for the spin-orbit conserving and changing manifolds, respectively.

originate from a combination of the $\mathrm{A}^{\prime}$ and $\mathrm{A}^{\prime \prime}$ potentials, and the strong forward peak is retained.

We have already established the importance of the $\mathrm{A}^{\prime}$ potential for the spin-orbit changing manifold, and Fig. 7 shows that the strong forward peak is a further signature of the $\mathrm{A}^{\prime}$ potential. The large impact parameters associated with the forward peak (see Fig. 5) suggest that Ar atoms approaching from the O-end (towards the $\mathrm{N}$-end) interact particularly efficiently with the electron once they reach the region of the extended width on the $\mathrm{N}$-side of the potential.

Based on the preceding analysis, it is tempting to attribute the forward scattered peak observed in the spin-orbit changing manifold exclusively to the concave shape of the repulsive core in the $\mathrm{A}^{\prime}$ PES. However, calculations run on truncated potentials, in which the attractive parts have been removed (see ESI $\dagger$ ), reveal that this conclusion is incorrect. As shown in Fig. S5 in the ESI, $\dagger$ the prominent forward peak is completely absent in the DCSs calculated on the truncated PESs (similar results are obtained using a hard shell QM model ${ }^{87}$ - see ESI $\dagger$ ). This is strong evidence that the attractive parts of the potential are essential for the appearance of the prominent forward peak in the spinorbit changing manifold.

The potential energy surfaces shown in Fig. 6 feature an attractive well on the side of the molecule. The well on the side of the $\mathrm{A}^{\prime}$ potential runs roughly parallel to and is deepest around the concave indentation of the repulsive wall. It is conceivable that high impact parameter trajectories approaching from the O-end of the molecule are efficiently funnelled through this attractive well and brought into close contact with the extended part of high electron density, so as to promote spinorbit excitation. The high impact parameter required for these kinds of trajectories results in minimal deflection of the Ar atom, as manifested in the sharp forward feature.

While the strong forward peak is most prominent for intermediate, even $\Delta j$ transitions, it is also present in the odd $\Delta j \leq 11$ transitions (see Fig. 4 and Fig. 5). However, due to the overall preference for $\mathrm{N}$-side/ $\mathrm{N}$-end collisions for the odd states, the forward peak is overshadowed by the main feature in the forward direction. The peak is perhaps most evident in the $-x$ geometry for $\Delta j=7,9$, where it is next to a minimum in the DCS. The prominence of the forward scattered feature in the even $\Delta j$ transitions is thus a combination of the characteristic topology of the $\mathrm{A}^{\prime}$ PES and the quantum interference effects which determine the overall preference for a specific orientation at a given $\Delta j$.

In order for the prominent forward scattered feature to appear, the unpaired electron must be lying in (or at least very close to) the triatomic plane defined by the NO molecule and the Ar atom. Given the shape of the $\mathrm{A}^{\prime}$ potential, this is the most efficient way for large impact parameter collisions to interact with the electron, and thus promote a spin-orbit excitation. For lower impact parameters (and larger scattering angles), the atom will be able to interact with the electron within a larger range of $\chi$ angles, and the dynamics are determined by contributions from both the $\mathrm{A}^{\prime}$ and $\mathrm{A}^{\prime \prime}$ potentials.

\section{Conclusions}

We have presented a combined experimental and theoretical study of the $\mathrm{NO}(\mathrm{X})$ spin-orbit changing transitions, $|\Omega|=1 / 2 \rightarrow$ $\left|\Omega^{\prime}\right|=3 / 2$, following oriented collisions with argon atoms. The experimentally measured differential and integral cross sections for the side-on geometry have been shown to be in good agreement with quantum mechanical calculations. As in previous studies, ${ }^{42,50,51}$ quantum interference effects, manifest as oscillations in the steric preference for $\mathrm{N}$-side or O-side collisions, have been observed.

The theoretical analysis and discussion has focused on the overall shift of the ISA in the spin-orbit changing manifold towards an $\mathrm{N}$-side/ $\mathrm{N}$-end preference and a prominent forward scattered peak, observed most intensely in the calculations for intermediate, even $\Delta j$ transitions in the O-end orientation. We have rationalized the shift of the ISA towards positive values with the higher unpaired electron density, and consequently 
more facile spin-orbit excitation, closer to the $\mathrm{N}$-side/N-end of the molecule, which leads to a competition between $\mathrm{N}$-side/ $\mathrm{N}$-end and (otherwise preferred) O-side/O-end collisions for even $\Delta j$ transitions. The ability to manipulate the initial relative orientation of the NO and the argon collision partners thus provides a direct probe of the unpaired electron's location and explains the importance of the $\mathrm{A}^{\prime}$ potential for scattering into the spin-orbit excited manifold. Our conclusions are consistent with the notion that spin-orbit excited transitions are primarily governed by the difference potential. ${ }^{78,79,81}$ The differences between the $\mathrm{A}^{\prime}$ and $\mathrm{A}^{\prime \prime}$ potentials are most significant on the $\mathrm{N}$-side, where the $\mathrm{A}^{\prime}$ potential is substantially wider than the $\mathrm{A}^{\prime \prime}$ potential, and this is where spin-orbit changing collisions most likely occur.

QM generalized deflection functions and infinite field calculations of the DCSs carried out on the $\mathrm{A}^{\prime}, \mathrm{A}^{\prime \prime}$, sum, and full potentials revealed that the prominent forward feature corresponds to high impact parameter collisions and originates from the specific topology of the $\mathrm{A}^{\prime}$ PES, an interplay between the repulsive region of high electron density near the N-side of the molecule and the attractive well nested into the concave region of the potential. We have argued that the attractive well efficiently funnels high impact parameter trajectories from the O-end, bringing the Ar atom into close contact with the unpaired electron on the $\mathrm{N}$-side of the molecule, and thus facilitating a change in spin-orbit quantum number.

Our study demonstrates how the ability to determine differential and integral cross sections for specific orientations of a diatomic molecule can yield very detailed insights into the scattering dynamics and the forces that govern them. Although the good agreement with the experimental measurements are further proof of the accuracy of the PESs used for the calculations, the angular resolution in our experiments is currently not high enough to resolve the sharp forward scattered feature predicted in the QM calculations (though there might be a hint of it in some of the images). However, in principle, it should be possible to combine our method of electric field orientation with a Stark decelerated crossed molecular beam setup; ${ }^{25,88-90}$ this would afford the angular resolution required to measure very sharp features in the DCSs. If such an experiment can be carried out successfully, it will provide a sensitive tool to test the accuracy of calculated potential energy surfaces and shed light on the associated scattering dynamics, potentially also in processes more complicated than simple diatom-atom scattering. In the specific case of our current work, the experimental confirmation of the prominent forward scattered feature in the DCSs for even $\Delta j$ transitions would offer an ultimate test for the accuracy of the $\mathrm{NO}+$ Ar potentials.

\section{Conflicts of interest}

There are no conflicts to declare.

\section{Acknowledgements}

F. J. A. and P. G. J. thank Prof. Enrique Verdasco for his help with the calculations. Funding by the UK EPSRC (to M. B. via
Programme Grant EP/L005913/1 and EP/T021675/1) and the Spanish Ministry of Science and Innovation (grant MINECO/ FEDER-PGC2018-096444-B-I00) is gratefully acknowledged. P. G. J. acknowledges funding by the Fundación Salamanca City of Culture and Knowledge (programme for attracting scientific talent to Salamanca).

\section{References}

1 P. Jankowski, A. R. W. McKellar and K. Szalewicz, Science, 2012, 336, 1147-1150.

2 P. Jankowski, L. A. Surin, A. Potapov, S. Schlemmer, A. R. W. McKellar and K. Szalewicz, J. Chem. Phys., 2013, 138, 084307.

3 G. Czakó and J. M. Bowman, J. Am. Chem. Soc., 2009, 131, 17534-17535.

4 C. Xiao, X. Xu, S. Liu, T. Wang, W. Dong, T. Yang, Z. Sun, D. Dai, X. Xu, D. H. Zhang and X. Yang, Science, 2011, 333, 440-442.

5 R. Otto, J. Ma, A. W. Ray, J. S. Daluz, J. Li, H. Guo and R. E. Continetti, Science, 2014, 343, 396-399.

6 M. Qiu, Z. Ren, L. Che, D. Dai, S. A. Harich, X. Wang, X. Yang, C. Xu, D. Xie, M. Gustafsson, R. T. Skodje, Z. Sun and D. H. Zhang, Science, 2006, 311, 1440-1443.

7 W. Dong, C. Xiao, T. Wang, D. Dai, X. Yang and D. H. Zhang, Science, 2010, 327, 1501-1502.

8 T. Wang, J. Chen, T. Yang, C. Xiao, Z. Sun, L. Huang, D. Dai, X. Yang and D. H. Zhang, Science, 2013, 342, 1499-1502.

9 J. B. Kim, M. L. Weichman, T. F. Sjolander, D. M. Neumark, J. Kłos, M. H. Alexander and D. E. Manolopoulos, Science, 2015, 349, 510-513.

10 M. Hapka, G. Chałasiński, J. Kłos and P. S. Żuchowski, J. Chem. Phys., 2013, 139, 014307.

11 T. Yang, J. Chen, L. Huang, T. Wang, C. Xiao, Z. Sun, D. Dai, X. Yang and D. H. Zhang, Science, 2015, 347, 60-63.

12 K. Stark and H. Werner, J. Chem. Phys., 1996, 104, 6515-6530.

13 D. Skouteris, D. E. Manolopoulos, W. Bian, H.-J. Werner, L.-H. Lai and K. Liu, Science, 1999, 286, 1713-1716.

14 S. Chefdeville, Y. Kalugina, S. Y. T. van de Meerakker, C. Naulin, F. Lique and M. Costes, Science, 2013, 341, 1094-1096.

15 C. Naulin and M. Costes, Int. Rev. Phys. Chem., 2014, 33, 427-446.

16 E. Lavert-Ofir, Y. Shagam, A. B. Henson, S. Gersten, J. Kłos, P. S. Żuchowski, J. Narevicius and E. Narevicius, Nat. Chem., 2014, 6, 332-335.

17 T. F. M. Luxford, T. R. Sharples, K. G. McKendrick and M. L. Costen, J. Chem. Phys., 2016, 145, 174304.

18 A. Klein, Y. Shagam, W. Skomorowski, P. S. Żuchowski, M. Pawlak, L. M. C. Janssen, N. Moiseyev, S. Y. T. van de Meerakker, A. van der Avoird, C. P. Koch and E. Narevicius, Nat. Phys., 2017, 13, 35-38.

19 C. L. Russell and D. E. Manolopoulos, Chem. Phys. Lett., 1996, 256, 465-473.

20 F. Lique, G. Li, H.-J. Werner and M. H. Alexander, J. Chem. Phys., 2011, 134, 231101. 
21 M. Tizniti, S. D. Le Picard, F. Lique, C. Berteloite, A. Canosa, M. H. Alexander and I. R. Sims, Nat. Chem., 2014, 6, 141-145.

22 S. M. Remmert, S. T. Banks, J. N. Harvey, A. J. Orr-Ewing and D. C. Clary, J. Chem. Phys., 2011, 134, 204311.

23 F. Wang, K. Liu and T. P. Rakitzis, Nat. Chem., 2012, 4, 636.

24 H. Guo and K. Liu, Chem. Sci., 2016, 7, 3992-4003.

25 S. N. Vogels, T. Karman, J. Kłos, M. Besemer, J. Onvlee, A. van der Avoird, G. C. Groenenboom and S. Y. T. van de Meerakker, Nat. Chem., 2018, 10, 435-440.

26 T. de Jongh, T. Karman, S. N. Vogels, M. Besemer, J. Onvlee, A. G. Suits, J. O. F. Thompson, G. C. Groenenboom, A. van der Avoird and S. Y. T. van de Meerakker, J. Chem. Phys., 2017, 147, 013918.

27 J. Kłos, Q. Ma, M. H. Alexander and P. J. Dagdigian, J. Chem. Phys., 2017, 146, 114301.

28 Y. Shagam, A. Klein, W. Skomorowski, R. Yun, V. Averbukh, C. P. Koch and E. Narevicius, Nat. Chem., 2015, 7, 921-926.

29 A. Bergeat, J. Onvlee, C. Naulin, A. van der Avoird and M. Costes, Nat. Chem., 2015, 7, 349-353.

30 F. Wang, J. Lin and K. Liu, Science, 2011, 331, 900-903.

31 F. Wang and K. Liu, J. Chem. Phys., 2016, 145, 144305.

32 F. Wang and K. Liu, J. Chem. Phys., 2016, 145, 144306.

33 H. Pan, F. Wang, G. Czakó and K. Liu, Nat. Chem., 2017, 9, 1175-1180.

34 W. E. Perreault, N. Mukherjee and R. N. Zare, Science, 2017, 358, 356-359.

35 W. E. Perreault, N. Mukherjee and R. N. Zare, Nat. Chem., 2018, 10, 561.

36 T. R. Sharples, J. G. Leng, T. F. M. Luxford, K. G. McKendrick, P. G. Jambrina, F. J. Aoiz, D. W. Chandler and M. L. Costen, Nat. Chem., 2018, 10, 1148-1153.

37 J. F. E. Croft, N. Balakrishnan, M. Huang and H. Guo, Phys. Rev. Lett., 2018, 121, 113401.

38 P. G. Jambrina, J. F. E. Croft, H. Guo, M. Brouard, N. Balakrishnan and F. J. Aoiz, Phys. Rev. Lett., 2019, 123, 043401.

39 B. Friedrich, D. R. Herschbach, J.-M. Rost, H.-G. Rubahn, M. Renger and M. Verbeek, J. Chem. Soc., Faraday Trans., 1993, 89, 1539-1549.

40 J. van Leuken, J. Bulthuis, S. Stolte and J. Snijders, Chem. Phys. Lett., 1996, 260, 595-603.

41 A. Gijsbertsen, H. Linnartz, C. A. Taatjes and S. Stolte, J. Am. Chem. Soc., 2006, 128, 8777-8789.

42 B. Nichols, H. Chadwick, S. D. S. Gordon, C. J. Eyles, B. Hornung, M. Brouard, M. H. Alexander, F. J. Aoiz, A. Gijsbertsen and S. Stolte, Chem. Sci., 2015, 6, 2202-2210.

43 J. Zou, S. D. S. Gordon, S. Tanteri and A. Osterwalder, J. Chem. Phys., 2018, 148, 164310.

44 S. D. S. Gordon, J. J. Omiste, J. Zou, S. Tanteri, P. Brumer and A. Osterwalder, Nat. Chem., 2018, 10, 1190-1195.

45 E. M. Jones and P. R. Brooks, J. Chem. Phys., 1970, 53, 55-58. 46 M. C. van Beek, G. Berden, H. L. Bethlem and J. J. ter Meulen, Phys. Rev. Lett., 2001, 86, 4001-4004.

47 A. Boca and B. Friedrich, J. Chem. Phys., 2000, 112, 3609-3619.

48 M. de Lange, M. Drabbels, P. Griffiths, J. Bulthuis, S. Stolte and J. Snijders, Chem. Phys. Lett., 1999, 313, 491-498.
49 M. Brouard, H. Chadwick, S. D. S. Gordon, B. Hornung, B. Nichols, F. J. Aoiz and S. Stolte, J. Chem. Phys., 2016, 144, 224301.

50 C. G. Heid, V. Walpole, M. Brouard, F. J. Aoiz and P. G. Jambrina, Nat. Chem., 2019, 11, 662-668.

51 V. Walpole, C. G. Heid, P. G. Jambrina, F. J. Aoiz and M. Brouard, J. Phys. Chem. A, 2019, 123, 8787-8806.

52 M. H. Alexander, J. Chem. Phys., 1999, 111, 7426-7434.

53 M. H. Alexander, J. Chem. Phys., 1999, 111, 7435-7439.

54 J. Kłos, G. Chałasiński, M. T. Berry, R. Bukowski and S. M. Cybulski, J. Chem. Phys., 2000, 112, 2195-2203.

55 P. Pajón-Suárez, G. Rojas-Lorenzo, J. Rubayo-Soneira and R. Hernández-Lamoneda, Chem. Phys. Lett., 2006, 421, 389-394.

56 H. Cybulski and B. Fernández, J. Phys. Chem. A, 2012, 116, 7319-7328.

57 Tutorials in Molecular Reaction Dynamics, ed. M. Brouard and C. Vallance, Royal Society of Chemistry, Cambridge, 2012.

58 C. Amiot, J. Mol. Spectrosc., 1982, 94, 150-172.

59 K. Schreel, J. Schleipen, A. Eppink and J. J. t. Meulen, J. Chem. Phys., 1993, 99, 8713-8722.

60 M. C. van Beek, J. J. ter Meulen and M. H. Alexander, J. Chem. Phys., 2000, 113, 637-646.

61 G. Paterson, S. Marinakis, J. Kłos, M. L. Costen and K. G. McKendrick, Phys. Chem. Chem. Phys., 2009, 11, 8804-8812.

62 L. Scharfenberg, K. B. Gubbels, M. Kirste, G. C. Groenenboom, A. van der Avoird, G. Meijer and S. Y. T. van de Meerakker, Eur. Phys. J. D, 2011, 65, 189-198.

63 G. Sarma, S. Marinakis, J. J. ter Meulen, D. H. Parker and K. G. McKendrick, Nat. Chem., 2012, 4, 985-989.

64 J. J. van Leuken, F. H. W. van Amerom, J. Bulthuis, J. G. Snijders and S. Stolte, J. Phys. Chem., 1995, 99, 15573-15579.

65 H. Kohguchi, T. Suzuki and M. H. Alexander, Science, 2001, 294, 832-834.

66 A. Gijsbertsen, H. Linnartz, G. Rus, A. E. Wiskerke, S. Stolte, D. W. Chandler and J. Kłos, J. Chem. Phys., 2005, 123, 224305.

67 X.-D. Wang, P. A. Robertson, F. J. J. Cascarini, M. S. Quinn, J. W. McManus and A. J. Orr-Ewing, J. Phys. Chem. A, 2019, 123, 7758-7767.

68 J. Brown, C. Kerr, F. Wayne, K. Evenson and H. Radford, J. Mol. Spectrosc., 1981, 86, 544-554.

69 C. J. Eyles, M. Brouard, H. Chadwick, B. Hornung, B. Nichols, C.-H. Yang, J. Kłos, F. J. Aoiz, A. Gijsbertsen, A. E. Wiskerke and S. Stolte, Phys. Chem. Chem. Phys., 2012, 14, 5403-5419.

70 C. J. Eyles, M. Brouard, H. Chadwick, F. J. Aoiz, J. Kłos, A. Gijsbertsen, X. Zhang and S. Stolte, Phys. Chem. Chem. Phys., 2012, 14, 5420-5439.

71 M. Brouard, H. Chadwick, S. D. S. Gordon, B. Hornung, B. Nichols, J. Kłos, F. J. Aoiz and S. Stolte, J. Chem. Phys, 2014, 141, 164306.

72 M. Brouard, S. D. S. Gordon, B. Nichols, V. Walpole, F. J. Aoiz and S. Stolte, Phys. Chem. Chem. Phys., 2019, 21, 14173-14185.

73 M. Brouard, H. Chadwick, S. D. S. Gordon, C. G. Heid, B. Hornung, B. Nichols, J. Kłos, P. G. Jambrina and F. J. Aoiz, Chin. J. Chem. Phys., 2020, 33, 217-233.

74 M. H. Alexander and S. Stolte, J. Chem. Phys., 2000, 112, 8017-8026. 
75 R. Uberna, R. D. Hinchliffe and J. I. Cline, J. Chem. Phys., 1996, 105, 9847-9858.

76 A. J. B. Eppink and D. H. Parker, Rev. Sci. Instrum., 1997, 68, 3477-3484.

77 D. W. Chandler and P. L. Houston, J. Chem. Phys., 1987, 87, 1445-1447.

78 M. H. Alexander, Chem. Phys., 1985, 92, 337-344.

79 M. H. Alexander, J. Chem. Phys., 1993, 99, 7725-7738.

80 R. N. Zare, Angular Momentum, Wiley-Interscience, New York, 1987.

81 M. H. Alexander, J. Chem. Phys., 1982, 76, 5974-5988.

82 P. G. Jambrina, M. Menéndez and F. J. Aoiz, Chem. Sci., 2018, 9, 4837-4850.

83 P. G. Jambrina, M. Menéndez, A. Zanchet, E. García and F. J. Aoiz, Phys. Chem. Chem. Phys., 2019, 21, 14012-14022.

84 HIBRIDON is a package of programs for the time-independent quantum treatment of inelastic collisions and photodissociation written by M. H. Alexander, D. E. Manolopoulos, H. Werner and B. Follmeg, with contributions by P. F. Vohralik, D. Lemoine, G. Corey, R. Gordon, B. Johnson, T. Orlikowski, A. Berning, A. D. Esposti, C. Rist, P. Dagdigian, B. Pouilly, G. van der Sanden, M. Yang, F. de Weerd, S. Gregurick and J. Kłos.

85 M. Brouard, S. D. S. Gordon, A. Hackett Boyle, C. G. Heid, B. Nichols, V. Walpole, F. J. Aoiz and S. Stolte, J. Chem. Phys., 2017, 146, 014302.

86 X. Zhang and S. Stolte, Chem. Phys., 2018, 514, 4-19.

87 M. Brouard, B. Hornung and F. J. Aoiz, Phys. Rev. Lett., 2013, 111, 183202.

88 J. Onvlee, S. N. Vogels, A. van der Avoird, G. C. Groenenboom and S. Y. T. van de Meerakker, New J. Phys., 2015, 17, 055019.

89 A. von Zastrow, J. Onvlee, D. H. Parker and S. Y. T. van de Meerakker, EPJ Techn. Instrum., 2015, 2, 11.

90 S. N. Vogels, Z. Gao and S. Y. T. van de Meerakker, EPJ Techn. Instrum., 2015, 2, 12. 